\title{
Emission Lines in the Far-UV and Extreme-UV Region
}

\author{
W. Zheng \\ Center for Astrophysical Sciences, Johns Hopkins University, Baltimore, \\ MD 21218, USA
}

\begin{abstract}
Recent observations with $H S T$ and $H U T$ have been made of the spectral region shortward of Ly $\alpha$ in AGN. Emission lines in this region, such as $\mathrm{O}$ VI $\lambda 1035$ and $\mathrm{NeV}$ III $\lambda 774$, are mainly produced by collisional excitation. Their strengths and the lack of other significant emission lines of higher excitation energy indicate a temperature of $\sim 6 \times 10^{4} \mathrm{~K}$ for the highly ionized gas in line-emitting clouds. Model calculations suggest that the strength of O VI and Ne VIII emission is correlated to the intensity of the high-energy photons above $100 \mathrm{eV}$. Therefore, these lines are useful diagnostics of the continuum shape in the soft X-ray range. The luminosity dependence of emission-line equivalent widths is more prominent for lines of higher ionization level, suggesting that the UV bump in AGN spectra may be weaker at higher luminosities.
\end{abstract}

\section{Introduction}

Our study of emission lines starts in the optical region, and gradually extends to the UV region and even beyond. A significant number of resonance atomic transitions fall in the UV region. Observations with $I U E$ over the past 18 years have provided a wealth of AGN spectra (Kinney, Rivolo, \& Koratkar 1990; Lanzetta, Turnshek, \& Sandoval 1992) that enable us to study Ly $\alpha$, C IV, and many other UV lines. In recent years, frequent IUE observations have been coordinated with ground-based and X-ray observations to monitor time variations of the continuum and emission lines, Ly $\alpha$ and CIV in particular. Such studies have established more accurate estimates of the size of the broad-line region in Seyfert galaxies and revealed the stratification in the region (Peterson 1993).

The properties of the lines below $1216 \AA$ have not been studied as well as Ly $\alpha$ and $\mathrm{C}$ IV. The $\mathrm{O}$ VI $\lambda 1035$ emission line was measured in the IUE spectra of medium-redshift quasars (Kinney et al. 1985). This line is a doublet, blended with Ly $\beta$. Recently, Laor et al. (1994) used high-resolution HST data to study the emission lines in four bright quasars. They were able to deblend Ly $\beta$ from $\mathrm{O}$ VI and found $\operatorname{Ly} \beta / \operatorname{Ly} \alpha=0.03-0.12$. Weak features such as $\operatorname{Ly} \gamma, \mathrm{C}$ III $\lambda 977, \mathrm{~S}$ VI $\lambda \lambda 933,945$, and $N$ III $\lambda 991$ have also been identified. Redshifted $\mathrm{O}$ vi emission is a common feature in the optical spectra of many high- $z$ quasars, but its profile suffers from severe Ly $\alpha$ forest absorption. An observational gap existed in the sub-Ly $\alpha$ region of many well-studied low-redshift Seyfert galaxies. 


\section{HUT Observations}

The Hopkins Ultraviolet Telescope (HUT, Davidsen 1993) is a 0.9-m telescope capable of spectroscopy between 850 and $1850 \AA$, with a resolution of $\sim 2.5 \AA$. It was flown during the Astro-1 mission between 1990 December 2-11. In March 1995 an improved version of the HUT made a second, 16-day flight on the Astro2 mission aboard the Space Shuttle Endeavour (Kruk et al. 1995). The HUT data base on more than 300 celestial targets includes far-UV spectra of about twenty Seyfert galaxies and quasars.

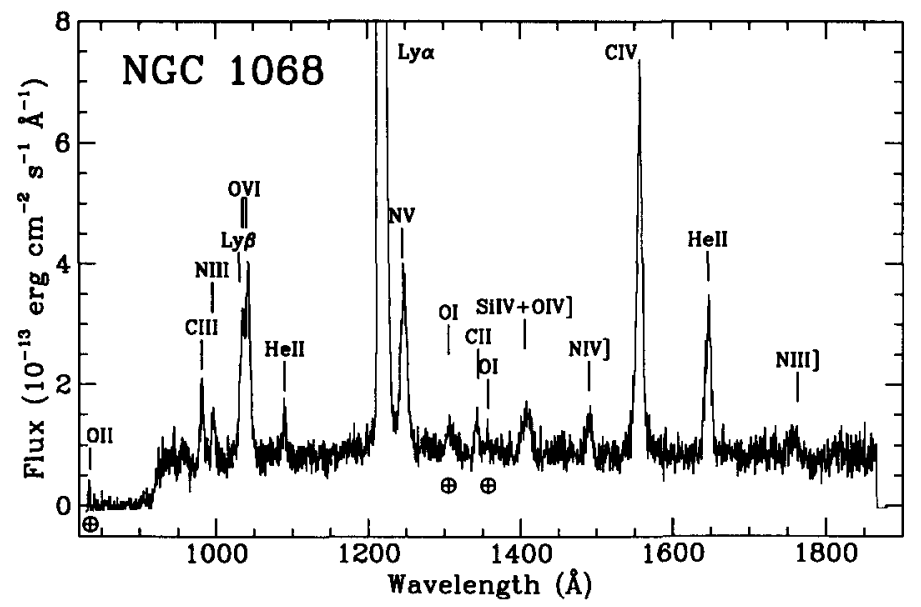

Figure 1. HUT spectrum of NGC 1068. Significant narrow emission lines are present. Airglow lines are marked with Earth symbols. Adopted from Kriss et al. (1992)

A good example of HUT data is the spectrum of the Seyfert 2 galaxy NGC 1068 (Kriss et al. 1992) shown in Fig. 1. The spectrum reveals strong lines of $\mathrm{C}_{\text {III }} \lambda 977, \mathrm{~N}_{\text {III }} \lambda$ 991, Ly $\beta, \mathrm{O}$ VI $\lambda \lambda 1032,1038$, and He II $\lambda 1085$. The ratios of $\left.\mathrm{C}_{111}\right] \lambda 1909$ to $\lambda 977$ and $\mathrm{N}$ III] $\lambda 1750$ to $\lambda 991$ imply temperatures exceeding $24,000 \mathrm{~K}$, much higher than those typically encountered in photoionized gas with abundant $\mathrm{C}^{+2}$ and $\mathrm{N}^{+2}$ populations.

Figure 2 shows the HUT spectra of Mrk 335 and Fairall 9. The two Seyfert 1 galaxies have different soft X-ray properties. Mrk 335 has a very strong soft Xray excess (Walter \& Fink 1993). Its O VI/C IV ratio of 0.58 is one of the highest among our candidates. Fairall 9 has a weak soft X-ray excess. Its O vi/C IV ratio is only 0.36 . There appears to be a correlation between the $\mathrm{O}$ VI strength and the soft X-ray intensity. Zheng, Kriss, \& Davidsen (1995) found that the objects with stronger $\mathrm{X}$-ray intensity (at $2 \mathrm{keV}$ ) exhibit stronger $\mathrm{O} \mathrm{VI}$ emission. Recent Astro-2 observations, including that of Fairall 9, further suggest that $\mathrm{O}$ VI strength is more closely related to the soft X-ray intensity below $1 \mathrm{keV}$. 


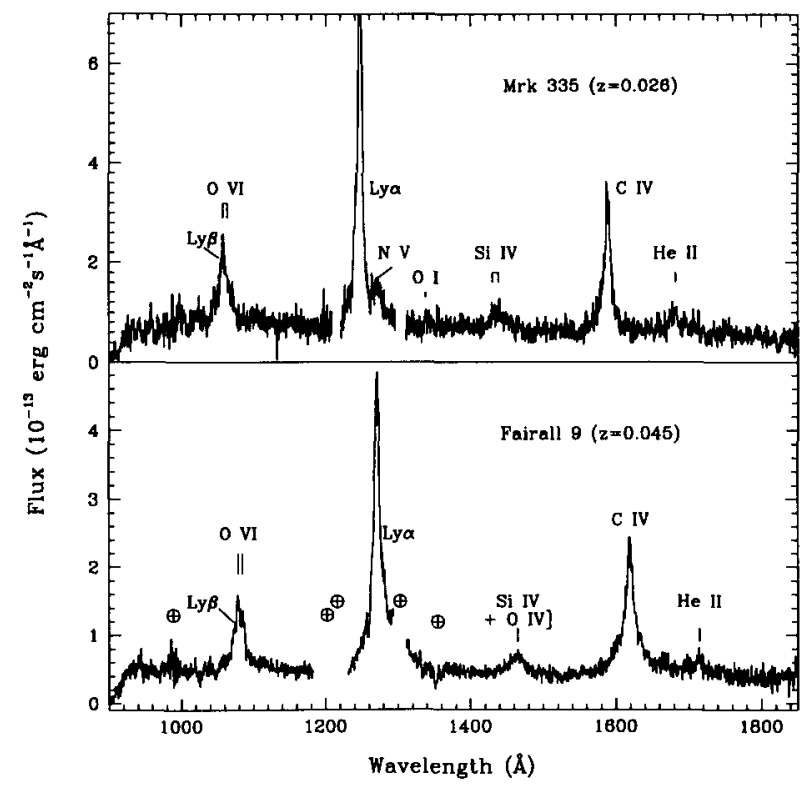

Figure 2. HUT spectra of Mrk 335 and Fairall 9. Airglow features are marked with Earth symbols.

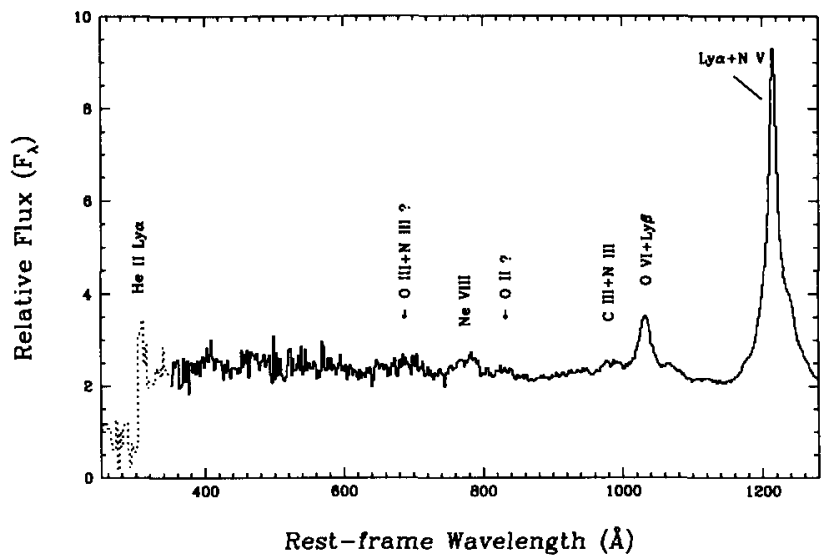

Figure 3. Quasar extreme-ultraviolet spectrum. The data above $350 \AA$ are from summed HST FOS spectra of high- $z$ quasars. The dotted curve displays the HUT spectrum of the quasar HS1700+64 below $350 \AA$ (Davidsen et al. 1996). A normalization is applied to connect the two curves. The sharp depression in flux below $\sim 300 \AA$ is due to intergalactic He II Ly $\alpha$ absorption. 


\section{HST Observations}

Hundreds of HST Faint Object Spectrograph (FOS) spectra reveal the sub-Ly $\alpha$ region of quasars of $z>0.2$. Figure 3 displays the spectral region below $1216 \AA$ in the rest frame. The curve above $350 \AA$ is a composite quasar spectrum (Zheng et al. 1996) based on the FOS spectra of quasars of $z>0.33$. There are no significant emission lines except for Ne viri $\lambda 774$ (Hamann, Zuo, \& Tytler 1995) below $1000 \AA$. This line has an exceptionally high ionization level and requires very strong soft X-ray emission to power it. The features near $690 \AA$ and $800 \AA$ are not certain due to the limited $S / N$ level. They may be due to the Bowen fluorescence mechanism (see below).

At the very short wavelength end, the HUT spectrum of the quasar HS $1700+64$ (Davidsen, Kriss, \& Zheng 1996) is normalized and displayed with a dotted curve. There is a prominent He II Ly $\alpha$ emission line at $304 \AA$, and its intensity relative to $\mathrm{Ly} \alpha$ is consistent with photoionization models. This line is produced by recombination and powered by high-energy photons of $E>54 \mathrm{eV}$. Note the blue wing of this line is suppressed by the absorption by the intergalactic medium. Abundant He II Ly $\alpha$ photons in the line-forming region lead to the Bowen fluorescence effect (Eastman \& MacAlpine 1985; Netzer, Elitzur, \& Ferland 1985). The $\mathrm{O}^{++}$and $\mathrm{N}^{++}$ions have transitions whose wavelengths coincide very closely with He II Ly $\alpha \lambda 304$. Theoretical estimates suggest that the $\mathrm{O}$ III/He II Ly $\alpha$ intensity ratio is of order 0.1 and is only weakly dependent on the ionizing flux. The $690 \AA$ feature is not present in every spectrum that covers the wavelengths between $650 \AA$ and $700 \AA$, so its identification is only suggestive.

\section{Discussion}

\subsection{Cloud Temperature}

A typical photoionization model (Kwan \& Krolik 1981) suggests that the highionization lines are formed in the ionized region where the temperature is of order $3 \times 10^{4} \mathrm{~K}$. Several UV metal lines are the major coolant for the region, keeping the temperature in a narrow range of thermal equilibrium, and therefore provide useful temperature estimates.

As shown in Table 1, these collisionally excited lines require a temperature $T \gtrsim 4 \times 10^{4} \mathrm{~K}$ for their presence. The collisional excitation rate is proportional to $\exp \left(-E_{e x c} / k T\right)$ where $E_{e x c}$ is the excitation energy (Osterbrock 1989). Only when the cloud temperature is comparable to the excitation temperature, does the collisionally excited line becomes significant. The EUV region contains a large number of resonance metal lines, and many of them have been identified as intervening absorption lines (Vogel \& Reimers 1995). The lack of emission lines below $750 \AA$ suggests that the temperature of the highly ionized gas in the broad-line region is not likely to exceed $6 \times 10^{4} \mathrm{~K}$.

\subsection{Effect of the Ionizing Continuum}

The intensity of each emission line depends on the number of photons above the line's ionization threshold. While the intensities of high-ionization lines are related to the continuum shape (Krolik \& Kallman 1988), they are also sensitive 
Table 1. Major FUV and EUV Emission Lines.

\begin{tabular}{lcc}
\hline \hline $\begin{array}{c}\text { Line } \\
(\AA)\end{array}$ & $\begin{array}{c}\text { Ionization } \\
\text { Energy }(\mathrm{eV})\end{array}$ & $\begin{array}{c}\text { Characteristic } \\
\text { Temperature }(\mathrm{K})\end{array}$ \\
\hline C IV $\lambda 1549$ & 8.0 & $4.0 \times 10^{4}$ \\
O VI $\lambda 1035$ & 12.0 & $6.0 \times 10^{4}$ \\
N III $\lambda 991$ & 12.5 & $6.3 \times 10^{4}$ \\
C III $\lambda 977$ & 12.7 & $6.4 \times 10^{4}$ \\
Ne VIII $\lambda 774$ & 16.0 & $8.1 \times 10^{4}$ \\
\hline
\end{tabular}

${ }^{\text {a }}$ The value at which $\exp \left(-E_{e x c} / k T\right)=0.1$ and the line strength starts to become significant.

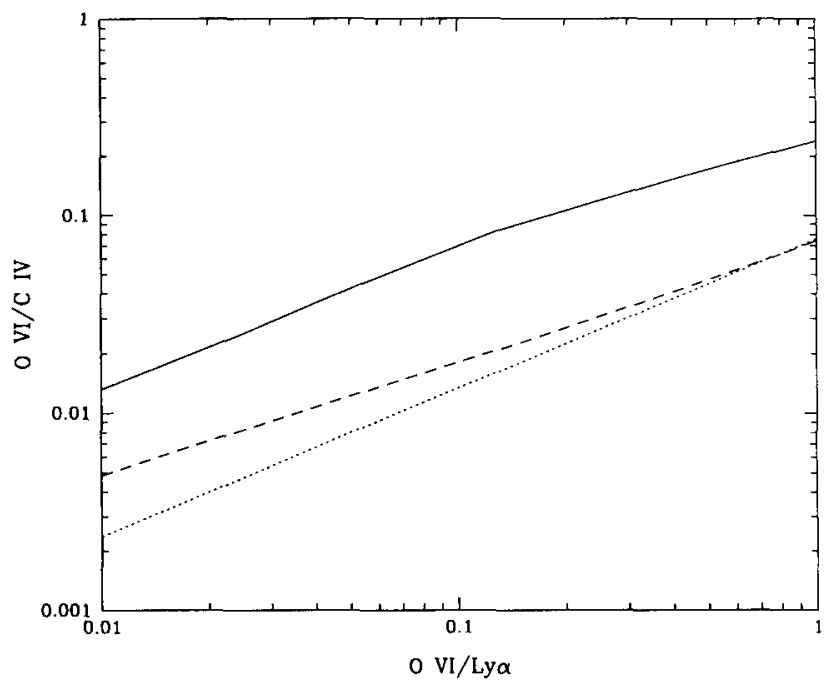

Figure 4. Theoretical line ratios produced with a power law plus different EUV bumps. Solid curve: With a bump centered at $100 \mathrm{eV}$; dotted curve: $50 \mathrm{eV}$, and dashed curve: $25 \mathrm{eV}$. 


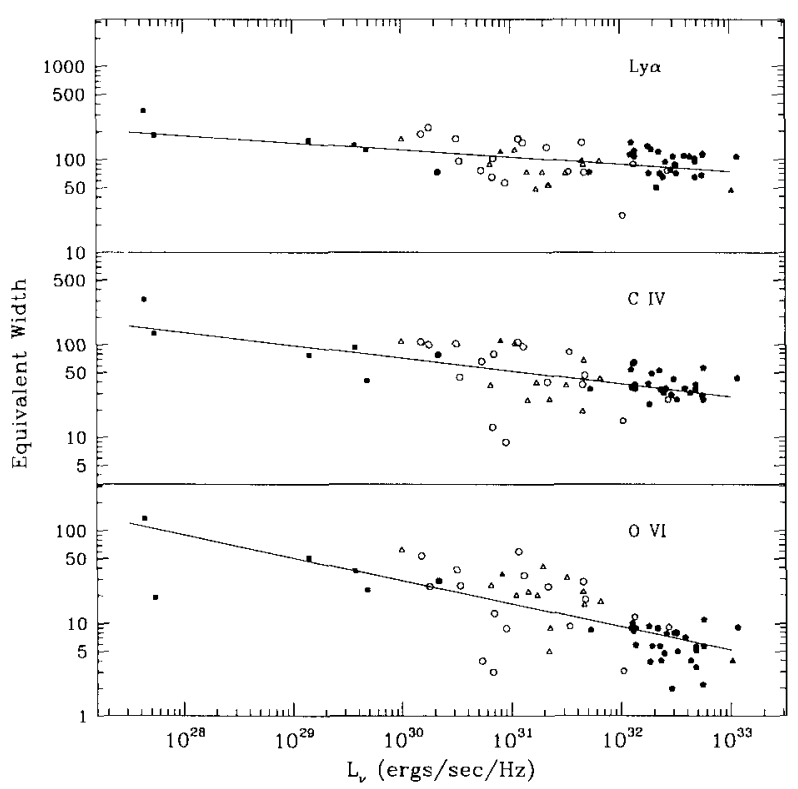

Figure 5. EW of Ly $\alpha, \mathrm{CIV}$, and O vi vs. specific luminosity of UV continuum $\mathrm{L}_{\nu}$, and their best fit. Squares: HUT data; circles: IUE data; triangles: HST data; pentagons: optical data. Solid symbols are for radio-quiet objects.

to the level of the ionizing flux. Namely, a stronger flux may mimic the effect of a harder ionizing continuum.

Take the example of $\mathrm{O}$ VI. It is a collisionally excited line and highly sensitive to the temperature. Would an ionizing source with abundant photons below $113 \mathrm{eV}$ (the ionization threshold of $\mathrm{O} \mathrm{VI}$ ) produce excessive heat in the line-forming region, thus boosting the OVI emission? A series of photoionization calculations are carried out using the code CLOUDY (Ferland 1993). The basic ionizing continuum is a power law of $F_{\nu} \propto \nu^{-2}$ between $1 \mathrm{eV}$ and $200 \mathrm{eV}$ and $F_{\nu} \propto \nu^{-0.7}$ between $200 \mathrm{eV}$ and $20 \mathrm{keV}$. Three models assume an additional EUV bump centered at 25,50 and $100 \mathrm{eV}$, respectively. The bump is assumed to be a Gaussian, with its width as $20 \%$ of the peak value. It is normalized so that its total energy is equal to that of the underlying power-law continuum. For each continuum model, several calculations are carried out with different ionization parameters for a fixed nucleon density of $10^{10} \mathrm{~cm}^{-3}$. As shown in Fig. 4, only the model with $100 \mathrm{eV}$ bump can produce both the O vi/Ly $\alpha$ and $\mathrm{O}$ VI/C IV ratios that are close to the observed values. The other two models produces over predict the CIV emission because of the bump at lower energies.

This example demonstrates that the number of photons with energy above $113 \mathrm{eV}$ dictates the temperature and volume of the region in which $\mathrm{O}$ vi emission 
is formed, and therefore it is possible to use multi-line ratios to diagnose the continuum shape in the EUV and soft X-ray band.

\subsection{Luminosity Dependence}

The intensities of high-ionization lines are known to exhibit an inverse luminosity dependence (Baldwin 1977; Kinney et al. 1990). Zheng, Fang, \& Binette (1992) found that such a relation is more significant for lines of higher ionization level. Zheng et al. (1995) found that, over five decades of luminosity, the Ovi equivalent width shows a very strong anti-correlation with luminosity (Fig. 5). Expressing such a correlation with $\log (E W)=A \log L_{U V}+$ constant, we find that, for Ly $\alpha, \mathrm{C} I V$, and O VI, the coefficient $A$ is $-0.076,-0.14$, and -0.25 respectively. Between Seyfert 1 galaxies and high- $z$ quasars the equivalent widths of Ly $\alpha, \mathrm{C}$ IV, and $\mathrm{O}$ VI decrease approximately by factors of 3,6 , and 25 , respectively. The significant change in high-ionization line intensity with luminosity is consistent with the assumption that the soft X-ray continuum between 0.1 and $1 \mathrm{keV}$ in high-luminosity quasars is weaker than that in low-luminosity AGNs.

Acknowledgments. I am grateful to G. A. Kriss and A.F. Davidsen for their comments and for allowing me to quote their published results. This work is supported in part by NASA contract NAS5-27000, and by grant AR4389.01-92A from the Space Telescope Science Institute, which is operated by the Association of Universities for Research in Astronomy, Inc., under NASA contract NAS5-26555.

\section{References}

Baldwin, J. A. 1977, ApJ, 214, 679.

Davidsen, A. F. 1993, Science, 259, 327.

Davidsen, A.F., Kriss, G.A., \& Zheng, W. 1996, Nature, 380, 47.

Eastman, R. G., \& MacAlpine, G. M. 1985, ApJ, 299, 785.

Ferland, G.J. 1993, Univ. Kentucky Dept. of Phys. \& Astron. Internal Report. Hamann, F., Zuo, L., \& Tytler, D. 1995, ApJ, 444, L69.

Kinney, A. L., Huggins, P. J., Bregman, J. N., \& Glassgold, A. E. 1985, ApJ, 291, 128.

Kinney, A. L., Rivolo, A. R., \& Koratkar, A. P. 1990, ApJ, 357, 338,

Kriss, G. A., Davidsen, A. F., Blair, W. P., Ferguson, H.C., \& Long, K. S. 1992, ApJ, 394, L37.

Krolik, J. H., \& Kallman, T. R. 1988, ApJ, 324, 714.

Kruk, J. W., Durrance, S.D., Kriss, G.A., Davidsen, A.F., Blair, W. P., Espey, B. R., \& Finley, D.S. 1995, ApJ, 454, L1.

Kwan, J., \& Krolik, J.H. 1981, ApJ, 250, 478.

Lanzetta, K. M., Turnshek, D. A., \& Sandoval, J. 1992, ApJS, 84, 109 .

Laor, A., Bahcall, J. N., Januzzi, B. T., Schneider, D. P., Green, R. F., \& Hartig, G. F. 1994, ApJ, 420, 110.

Netzer, H., Elitzur, M., \& Ferland, G.J. 1985, ApJ, 299, 752. 
Osterbrock, D.E. 1989, Astrophysics of Gaseous Nebulae and Active Galactic Nuclei (Mill Valley: University Science Books).

Peterson, B. M. 1993, PASP, 105, 247.

Vogel, S., \& Reimers, D. 1995, A\&A, 294, 377.

Walter, R., \& Fink, H.H. 1993, A\&A, 274, 105.

Zheng, W., Fang, L. Z., \& Binette, L. 1992, ApJ, 392, 74.

Zheng, W., Kriss, G. A., \& Davidsen, A.F. 1995, ApJ, 440, 606.

Zheng, W., Kriss, G.A., Telfer, R. C., Grimes, J.P., \& Davidsen, A.F. 1996, ApJ, submitted. 